\title{
Acrylamide precipitation polymerization in a continuous flow reactor: an in situ FTIR study reveals kinetics
}

\author{
Pascal Fandrich $^{1} \cdot$ Lars Wiehemeier $^{1} \cdot$ Maxim Dirksen $^{1} \cdot$ Oliver Wrede $^{1} \cdot$ Tilman Kottke $^{1} \cdot$ Thomas Hellweg $^{1}$
}

Received: 25 August 2020 / Revised: 8 September 2020 / Accepted: 29 September 2020 / Published online: 20 November 2020 (C) The Author(s) 2020

\begin{abstract}
In this work, we present a combination of a continuous flow reactor with in situ monitoring of the monomer conversion in a precipitation polymerization. The flow reactor is equipped with a preheating area for the synthesis of thermoresponsive microgels, based on $N$-isopropylacrylamide (NIPAM). The reaction progress is monitored with in situ FTIR spectroscopy. The monomer conversion at defined residence times is determined from absorbance spectra of the reaction solutions by linear combination with reference spectra of the stock solution and the purified microgel. The reconstruction of the spectra appears to be in good agreement with experimental data in the range of 1710 to $1530 \mathrm{~cm}^{-1}$, in which prominent absorption bands are used as probes for the monomer and the polymer. With increasing residence time, we observed a decrease in intensity of the $v(\mathrm{C}=\mathrm{C})$ vibration, originating from the monomer, while the $v(\mathrm{C}=\mathrm{O})$ vibration is shifted to higher frequencies by polymerization. Differences between the determined inline conversion kinetics and offline growth kinetics, determined by photon correlation spectroscopy (PCS), are discussed in terms of diffusion and point to a crucial role of mixing in precipitation polymerizations.
\end{abstract}

Keywords Microgels · NIPAM · Continuous flow reactor · in situ FTIR · Precipitation polymerization · Growth kinetics

\section{Introduction}

In the past few years, the continuous synthesis of smart microgels has attracted more and more attention, because of the convenient up-scaling of production. Smart microgels are polymer particles, typically swollen with a solvent, which can respond to an external stimulus by a reversible volume phase transition (VPT) [1-5]. The size of these colloidal gels ranges from a few tens of $\mathrm{nm}$ to approximately $1 \mu \mathrm{m}$. The ability to respond to an external stimulus, such as a change of temperature [6], $\mathrm{pH}$ value [7], pressure [8], solvent [9], or ionic strength [10], makes microgels interesting candidates for a broad range of applications. The response typically results in a change of the particles size,

Electronic supplementary material The online version of this article (https://doi.org/10.1007/s00396-020-04762-w) contains supplementary material, which is available to authorized users.

Thomas Hellweg

thomas.hellweg@uni-bielefeld.de

1 Dep. of Chemistry, Physical and Biophysical Chemistry, Bielefeld University, Universitätsstr. 25, 33615Bielefeld, Germany which is of great interest in various fields, like controlled catalysis [11, 12], biocatalysis [13, 14], sensor design [15-18], drug delivery $[19,20]$, microreactors [21], smart membrane fabrication [22, 23], surface coating [24, 25] and microfluidics [26-28]. The properties of microgels can be influenced by copolymerization [29] or by architecture [30-33].

The best studied representative of thermoresponsive microgels is based on $N$-isopropylacrylamide (NIPAM), cross-linked with $N, N^{\prime}$-methylenebis(acrylamide) (BIS). Above a temperature of approximately $32{ }^{\circ} \mathrm{C} \operatorname{poly}(N$ isopropylacrylamide) (PNIPAM) microgels show a VPT in water by releasing solvent out of the polymer network, usually within some milliseconds [8]. Batch syntheses of PNIPAM-based microgels via precipitation polymerization are well established. In such a synthesis, a free radical polymerization must be performed above the volume phase transition temperature (VPTT) of the polymer to form microgel particles. To tailor the properties of microgels in precipitation polymerizations, it is of major importance to investigate the microgel formation in detail. Considering in situ monitoring, three stages of the reaction progress are of great interest (Fig. 1).

Prior to initiation of the reaction, only the monomer and the cross-linker are present in the solution. After addition 


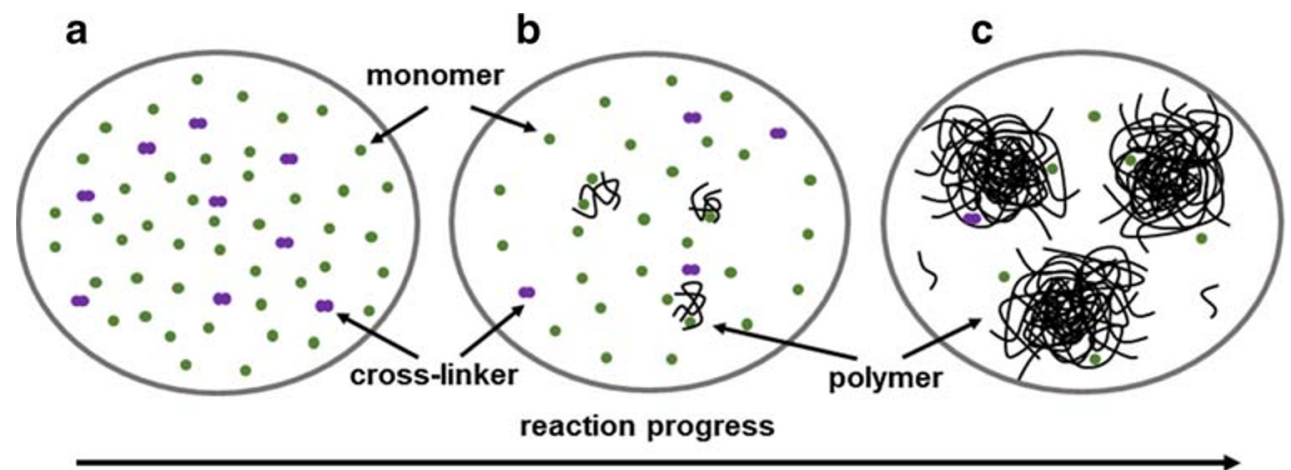

Fig. 1 The different stages in a precipitation polymerization can be divided in three characteristic polymerization steps. The stock solution (a) consists of dissolved monomer and cross-linker. The reaction starts by addition of a radical initiator, leading to formation of oligomers and

of a thermally unstable initiator, the precipitation polymerization is started and small oligomers and precursor particles are formed. During the reaction progress, the particles start to grow by radical polymerization and aggregation of oligomers in the particles. The final reaction solution consists of fully grown particles, with few monomers and small oligomers left [34]. In established batch syntheses the batch is preheated to the required reaction temperature before the reaction is initiated by addition of a radical initiator, often predissolved in a small volume with respect to the total volume [35-38].

The idea of a continuous production of thermoresponsive microgels has gained popularity, due to the great potential concerning production scale-up, screening and in situ monitoring [39]. Recent work shows that a continuous flow synthesis is a suitable method to synthesize thermoresponsive microgels and points out that mixing is of great importance for narrow particle size distributions [40]. In this context, mixing inside continuous flow reactors is very challenging, as static mixers not only lead to fluid mixing, but also tend to promote fouling, coalescence, or clogging when polymer films form onto the surface. Therefore, many of the continuous flow setups in literature are equipped with a mixer right before the reactor to mix the initiator with the monomer solution, before heating to the desired reaction temperature in the reactor [41, 42].

A combination with in situ monitoring of the reaction progress is of great interest to study defined stages of the microgel formation over a long period of time. So far, FTIR spectroscopy has been used as a non-invasive method to study the phase behavior in thermoresponsive acrylamides on a molecular level by analyzing the vibrational modes of the amide in $\mathrm{D}_{2} \mathrm{O}[43,44]$ and $\mathrm{H}_{2} \mathrm{O}$ [45]. Furthermore, the role of the polymer backbone [46] and the isopropyl groups $[47,48]$ in microgels during phase transition was studied with FTIR spectroscopy. In the context of inline monitoring, prominent vibrations in acrylamides have been collapsed precursor particles (b). The particles start to grow until they reach their full size with few, unreacted monomer and small oligomers left in the solution (c)

used as probes for monomer conversion in batch syntheses, using Raman spectroscopy [49]. Moreover, Virtanen et al. [50] studied the microgel formation by in situ SANS in a stopped-flow setup.

To the best of our knowledge, a combination of a continuous flow setup with in situ monitoring of a precipitation polymerization by FTIR spectroscopy has not been established yet. In this work, we present a continuous flow synthesis of thermoresponsive microgels in a flow reactor with preheating area and subsequent in situ monitoring of the polymerization progress by inline FTIR spectroscopy. We show that a combination of continuous flow syntheses and in situ monitoring is a convenient approach for continuous process control and screening of microgel syntheses. The growth kinetics of the microgels are derived from the hydrodynamic radii $R_{h}$, as obtained by PCS $[51,52]$. The monomer conversion is determined from FTIR spectra by using characteristic vibrational modes of the monomer and the polymer as probes. With our approach, we introduce a new concept to study defined stages of microgel formation over an arbitrary period of time.

\section{Materials and methods}

\section{Materials and solution preparation}

$N$-isopropylacrylamide (NIPAM) (> 98\%; TCI, Eschborn, Germany) was recrystallized from $n$-hexane. $N, N^{\prime}$-methylenebis(acrylamide) (BIS) (99\%; Sigma-Aldrich, St. Louis, USA), ammonium persulfate (APS) (> 98\%; Sigma-Aldrich, St. Louis, USA) and deuterium oxide $\left(\mathrm{D}_{2} \mathrm{O}\right)(99.9 \%$; Deutero, Kastellaun, Germany) were used as received. NIPAM (11.55 mmol, $79.4 \mathrm{mM})$ and BIS $(0.58 \mathrm{mmol}$, $5.0 \mathrm{~mol} \%$ to NIPAM) were dissolved in deuterium oxide $(145.5 \mathrm{~mL})$ in a three-necked flask and purged with nitrogen under stirring for $90 \mathrm{~min}$. After purging, the 
stock solution was degassed under vacuum and stirring for $30 \mathrm{~min}$. APS $(0.202 \mathrm{~g}, 0.89 \mathrm{mmol})$ was dissolved in $\mathrm{D}_{2} \mathrm{O}(10.0 \mathrm{~mL})$ and purged with nitrogen for $45 \mathrm{~min}$ under stirring. The concentrations were adjusted to the used flow conditions. The reaction solution always had a final NIPAM concentration of $77 \mathrm{mM}$.

\section{Setup}

Two low-pressure syringe pumps (neMESYS 290N, gear box translation: 14:1, Cetoni GmbH, Korbussen, Germany), equipped with two $10 \mathrm{~mL}$ glass syringes (Hamilton Company, Reno, USA) were used to pump the monomer solution into the reactor (Fig. 2). For a continuous pumping procedure, the cross-flow option in the software QMix Elements (version: 20191024, Cetoni, Korbussen, Germany) was used. The duration of the cross-flow overlap was set to $10 \mathrm{~s}$. With a third low-pressure syringe pump (neMESYS 290N, 29:1), equipped with a $10 \mathrm{~mL}$ glass syringe (Hamilton Company, Reno, USA), the initiator solution was injected. Further information about the used flows is shown in Table 1.

The flow reactor was a hollowed out aluminum cylinder $(150 \times 120 \times 90 \mathrm{~mm}$, wall thickness: $10 \mathrm{~mm})$, covered by a PVC box $(170 \times 140 \times 110 \mathrm{~mm}$, wall thickness: $10 \mathrm{~mm}$ ) for thermal insulation. The reaction took place in a tube with an inner diameter of $1.65 \mathrm{~mm}$. The tube consisted of an inner wall of $0.4 \mathrm{~mm}$ of poly(ethylene-2,5furanoate) (PEF) and an outer wall of $0.4 \mathrm{~mm}$ of polyvinylidene fluoride (PVDF). As mentioned before, the stock solution was continuously pumped into the reactor with a cross-flow procedure. The reactor was connected with a Haake P5 circulating bath with a Haake DC10 thermo controller (Thermo Fisher Scientific Inc., Massachusetts, USA) and heated up with water to $70^{\circ} \mathrm{C}$. While pumped into the reactor, the stock solution was preheated in the preheating area for at least $5 \mathrm{~min}$ to reach the reaction temperature. Subsequently, the preheated solution reached a T-junction with an inner diameter of $2 \mathrm{~mm}$, where the initiator solution was injected through a cannula with an inner diameter of $0.400 \mathrm{~mm}$. The reaction solution was then transferred through the reaction zone with the desired length (see Table 1). The received reaction solution was directly pumped through a flow cell to perform in situ FTIR experiments. Subsequently, the solution was quenched in a 77 mM aqueous solution of 4-methoxyphenol (99\%; SigmaAldrich, St. Louis, USA). The quenched microgels were purified by four successive centrifugation, decantation and redispersion steps in purified water $\left(20000 \mathrm{rpm}, 20^{\circ} \mathrm{C}, 1 \mathrm{~h}\right.$, 70Ti; Beckman Coulter, Brea, USA).

\section{Photon correlation spectroscopy}

Temperature-dependent PCS measurements were performed at a fixed angle of $45^{\circ}$ to obtain swelling curves of the purified microgels. A HeNe-laser $(632.8 \mathrm{~nm}, 21 \mathrm{~mW}$, Thorlabs, Newton, USA) was used in combination with a 6010 multiple tau digital autocorrelator (ALV, Langen, Germany) and an SO-SIPD single photon detector (ALV, Langen, Germany). The highly diluted sample was inserted in a decalin bath and measured in a range of 10 to $60^{\circ} \mathrm{C}$ (C25P, Thermo Haake, Karlsruhe, Germany). The sample was equilibrated for $25 \mathrm{~min}$ at each temperature. The hydrodynamic radius $R_{h}$ can be calculated from the StokesEinstein equation (Eq. 1).

$D_{T}=\frac{k_{B} T}{6 \pi \eta R_{h}}$

The Stokes-Einstein equation describes the translational diffusion coefficient $D_{T}$ considering the Boltzmann constant $k_{\mathrm{B}}$, the temperature $T$, the dynamic viscosity of the solvent $\eta$ and the hydrodynamic radius $R_{h} . D_{T}$ is described by the
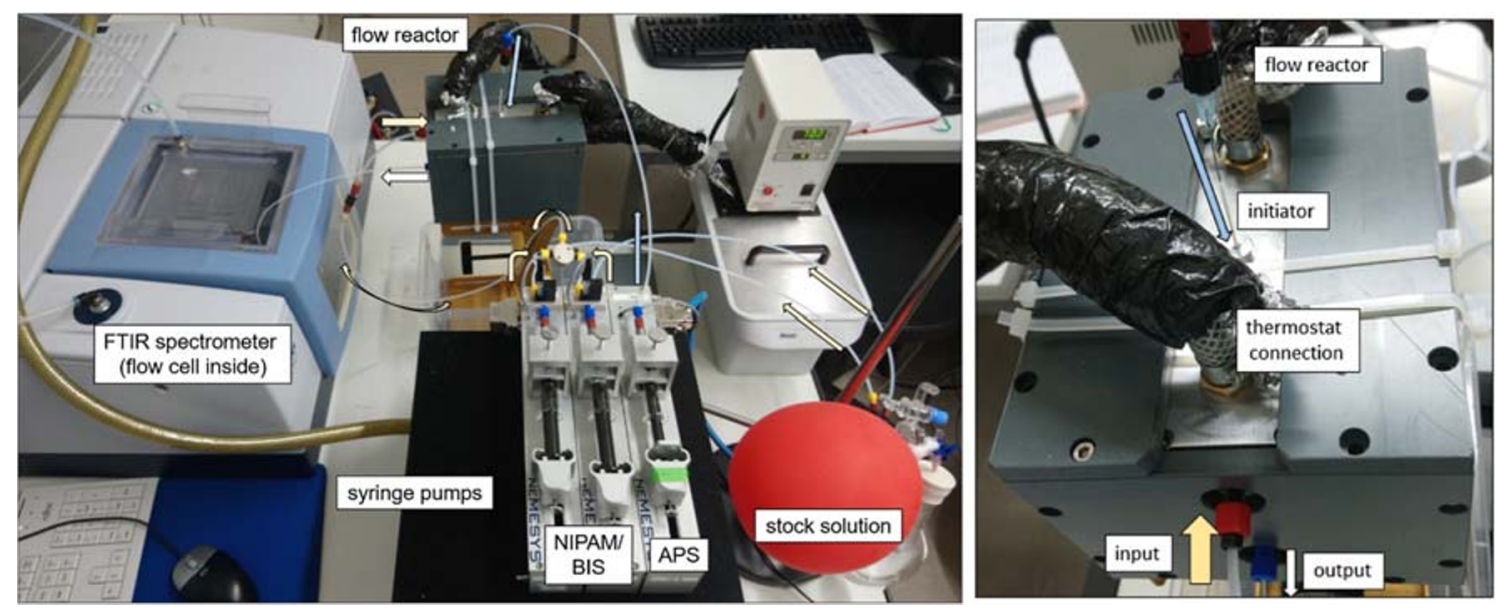

Fig. 2 Continuous flow setup for in situ measurements in a FTIR spectrometer 
Table 1 Detailed conditions for the adjusted residence times $t_{\mathrm{r}}$ inside the reaction zone. The flow ratio between flow stock $_{\text {and flow }}$ APS was set to $97: 3$

\begin{tabular}{lllll}
\hline $\begin{array}{l}t_{\mathrm{r}} \\
(\mathrm{min})\end{array}$ & $\begin{array}{l}\text { length } \\
(\mathrm{cm})\end{array}$ & $\begin{array}{l}\text { flow }_{\text {total }} \\
\left(\mathrm{mL} \mathrm{h}^{-1}\right)\end{array}$ & $\begin{array}{l}\text { flow } \\
\left(\mathrm{mL} \mathrm{h}^{-1}\right)\end{array}$ & $\begin{array}{l}\text { flow } \\
\left(\mu \mathrm{L} \mathrm{h}^{-1}\right)\end{array}$ \\
\hline 5 & 80 & 20.527 & 19.911 & 616 \\
6 & 80 & 17.106 & 16.593 & 513 \\
7 & 80 & 14.662 & 14.222 & 440 \\
8 & 80 & 12.830 & 12.445 & 385 \\
10 & 80 & 10.264 & 9.956 & 308 \\
15 & 600 & 51.318 & 49.778 & 1540 \\
30 & 600 & 25.659 & 24.889 & 770 \\
45 & 600 & 17.106 & 16.593 & 513 \\
60 & 600 & 12.830 & 12.445 & 385 \\
\hline
\end{tabular}

ratio of the mean relaxation rate $\bar{\Gamma}$ and the scattering vector $q$ (Eq. 2).

$D_{T}=\frac{\bar{\Gamma}}{q^{2}}$

The scattering vector $q$ is dependent on the wavelength $\lambda$, the refractive index of the solvent $n$ and the scattering angle $\theta$ (Eq. 3).

$q=|\mathbf{q}|=\frac{4 \pi n}{\lambda} \sin \left(\frac{\theta}{2}\right)$

The obtained data were analyzed with the CONTIN program by Laplace inversion [53].

\section{Static light scattering}

In addition to the PCS measurements the sample of the fully grown microgels $(60 \mathrm{~min})$ was measured by static light scattering. The setup was equipped with a cw-Laser (532.0 nm, Compass 315M-100 DPSS, Coherent Inc., Santa Clara, USA), a multiple tau digital correlator (ALV-7004, ALV-GmbH, Langen, Germany), a goniometer system (ALV/CGS-3, ALV-GmbH, Langen, Germany) equipped with a KP56KM1-501 step motor and a photon counting module (SPCM CD 3296 H, Excelitas Technologies Inc., Dumberry, Canada). The SLS measurements were performed at $50^{\circ} \mathrm{C}$ in a range between 30 and $140^{\circ}$ with an increment of $1^{\circ}$. Every angle was measured five times for $30 \mathrm{~s}$. Water and toluene were measured in the same way to obtain a normalized scattering intensity $I_{\text {norm }, \theta}$ (Eq. 4).

$I_{\text {norm }, \theta}=\frac{I_{\text {sample }, \theta}-I_{\mathrm{H}_{2} \mathrm{O}, \theta}}{I_{\text {toluene }, \theta}} R_{\text {toluene }}\left(\frac{n_{\mathrm{H}_{2} \mathrm{O}}}{n_{\text {toluene }}}\right)^{2}$

In Eq. $4 I_{\text {norm, } \theta}$ depends on the scattering intensity of the sample $I_{\text {sample }, \theta}$, the solvent $I_{\mathrm{H}_{2} \mathrm{O}, \theta}$ and toluene $I_{\text {toluene, } \theta}$, as well as the Rayleigh ratio $R_{\text {toluene }}$ and the refractive indices of water $n_{\mathrm{H}_{2} \mathrm{O}}$ and toluene $n_{\text {toluene. The scattering profile }}$ was fitted with a model-dependent hard-sphere fit using SASVIEW [54]. The model describes the scattered intensity $I(q)$ as a function of the volume fraction $\phi$, the volume of the scatterer $V$, the radius of the sphere $r$, the background $b$, the scattering vector $q$ and the scattering length difference between scatterer and the solvent $\Delta \rho$ (Eq. 5).

$I(q)=\frac{\phi}{V}\left(\frac{3 V(\Delta \rho)(\sin (q r)-q r \cos (q r))}{(q r)^{3}}\right)^{2}+b$

The radius of gyration $R_{g}$ was determined by a Guinier analysis. The data from SLS were fitted linearly up to $q R_{g}<1$ in relation to the maximum $q$-value of the fitting region (Eq. 6).

$\ln \left(I_{\text {norm }}(q)\right)=\ln \left(I_{\text {norm }}(0)\right)-\frac{\left(R_{g} q\right)^{2}}{3}$

The radius of gyration $R_{g}$ is defined by the slope of the linear behavior in Eq. 6 .

\section{Atomic force microscopy}

AFM measurements were used to investigate the structure and size distribution of single adsorbed particles in the dried state at room temperature. The measurements were performed with a DI Nanoscope IIIa (Digital Instruments, now Bruker, Karlsruhe, Germany) mounted on a Zeiss Axiovert 135 inverted microscope (Carl Zeiss Microscopy $\mathrm{GmbH}$, Jena, Germany) in semi-contact mode using Budget Sensors (Innovative Solution Bulgaria Ltd., Sofia, Bulgaria) Al-Reflex Tap300Al-G cantilevers with a tip radius of $<10 \mathrm{~nm}$, a resonance frequency of about $300 \mathrm{kHz}$ and a spring constant of $40 \mathrm{~N} / \mathrm{m}$. For the sample preparation a silicon wafer (Siegert Wafer $\mathrm{GmbH}$, Aachen, Germany) was cleaned with ethanol (HPLC grade) and subsequently in a plasma cleaner (Zepto, Diener Electronics $\mathrm{GmbH}$, Ebhausen, Germany). Afterwards, the cleaned wafer was spin-coated with $0.1 \mathrm{~mL}$ of a PEI-solution $(0.25 \mathrm{wt} \%)$ and then with a highly diluted microgel suspension. The resulting images were analyzed with GWYDDION [55] and Image J [56].

\section{FTIR spectroscopy}

In situ FTIR experiments were performed in a flow cell (Omni-Cell, Specac Ltd., Orpington, UK) with $\mathrm{CaF}_{2}$ windows (Korth Kristalle GmbH, Kiel, Germany). A PTFE spacer with a slit was placed between the windows (thickness: $0.1 \mathrm{~mm}$, width: $2 \mathrm{~mm}$, length: $4 \mathrm{~cm}$ ). The measurements were performed in a Tensor 27 spectrometer with the software OPUS 7.5 (Bruker, Ettlingen, Germany). The spectra were taken with at least 256 scans in a spectral 
region between 0 and $7900 \mathrm{~cm}^{-1}$ at a spectral resolution of $2 \mathrm{~cm}^{-1}$ and detected with a DTGS detector. A zero-filling factor of four was used for the Fourier transformation. The chamber of the spectrometer was continuously purged with dried air (Parker Hannifin, model 75-62, Cleveland, USA). A spectrum of $\mathrm{D}_{2} \mathrm{O}$ was recorded directly before each experiment as a reference spectrum. The spectra were corrected to compensate water vapor. The respective difference absorbance spectra were generated by subtraction of the solvent spectrum from the sample spectrum, and were baseline-corrected with a polynomial rubber band correction. The residence time between the reactor and the flow cell was kept as short as possible, though the solutions were investigated below the VPTT to avoid significant influences of the temperature on the shape and the area of the amide I vibration in the polymer (see SI: Fig. 1).

\section{Calculations of spectra}

Quantum chemical calculations of deuterated NIPAM and PNIPAM were performed with Gaussian03W [57]. The geometry optimization was performed with a density functional theory approach. A basis set of $6-311 \mathrm{G}+(2 \mathrm{~d}, \mathrm{p})$ with a B3LYP functional was used. The calculated spectra were scaled with a factor of 0.96 and broadened with Lorentzians using a full width at half maximum of $14 \mathrm{~cm}^{-1}$.

\section{Results and discussion}

\section{Design of a flow reactor with preheating area}

We developed a flow reactor that enables an in situ investigation of the reaction progress during the continuous flow synthesis of smart acrylamide-based microgels. To prevent fouling, i.e., a deposition of microgel films on the inside of the reactor, we chose a tubing made of poly(ethylene-2,5-furanoate) (PEF) on the inside with a polyvinylidene fluoride (PVDF) coating on the outside to prevent the solution from an uptake of oxygen and therefore an inhibition of the reaction. Since polymers, like PVDF, have a much lower heat conductivity than steel $[58,59]$ and to transfer the established protocol of batch syntheses to a continuous setup, we equipped the flow reactor with a preheating area to heat the stock solution up to the required reaction temperature before initiation (Fig. 3). For temperature control of both, the preheating area and the reaction zone, the whole reactor was submerged in a temperature-controlled water bath.

The preheated solution was pumped into a T-junction, where the dissolved initiator was added in a flow ratio of 3:97, with respect to the monomer flow. The final concentration of the resulting reaction solution was comparable to common batch concentrations in literature [60]. After initiation, the reaction solution was pumped through the reaction zone. The residence time in the reaction zone was adjusted by the total flow rate and the length of the tubing. With flow rates ranging from 10.3 to $51.3 \mathrm{~mL} \mathrm{~h}^{-1}$ and tube lengths of 80 and $600 \mathrm{~cm}$, residence times between 5 and 60 min were accessible (for more details, see Table 1). For all flow conditions, the temperature inside the Tjunction reached approximately $70^{\circ} \mathrm{C}$, guaranteeing stable reaction conditions (see SI: Fig. 2). To enable a high volume throughput, the stock solution was continuously dispensed by two syringe pumps, connected to a reservoir. Dispensing and aspirating were performed alternately. In combination with an autosampler (see SI: Fig. 3), continuous runs with durations of $>10 \mathrm{~h}$ could be realized. The reservoir can be refilled during the synthesis for experiments of arbitrary length. In the experiments performed in this work, the total duration was only limited by the volume of the syringe with the initiator solution. However, the setup may also be operated with a fourth syringe pump and a refillable reservoir for the initiator, which makes it suitable for essentially infinite operation.

To reduce pressure drops during a switch between two syringes, the pumps were synchronized by a cross-flow mode, to perform a smooth fade between the streams (see SI: Fig. 4,5). During preparation, the stock solution was purged with nitrogen to remove oxygen and afterwards degassed under vacuum with sonication to prevent gas bubble formation, which would lead to discontinuous flows and concentration variations in the flow cell. After leaving the reaction zone, the suspension was pumped through a flow cell, installed in an FTIR spectrometer for inline investigations. Afterwards, the reaction solution was quenched in a $p$-methoxyphenol solution, preserving the particles at their current reaction state for offline analysis. The time deviation caused by the additional duration from the reactor to the spectrometer and subsequently to the quenching solution was kept as short as possible and was taken into account in the following evaluation.

\section{Microgel characterization}

To characterize the microgels produced in the flow reactor after different residence times, we applied photon correlation spectroscopy (PCS), atomic force microscopy (AFM) and static light scattering (SLS).

In Fig. 4, the hydrodynamic radii of the purified microgels $R_{h}$, obtained from PCS measurements show a characteristic swelling behavior for PNIPAM/BIS microgels, between 10 and $60^{\circ} \mathrm{C}$. All microgels with a residence time between 5 and $60 \mathrm{~min}$ show a phase transition at approximately $33^{\circ} \mathrm{C}$, whereas the size ranges from 218 to $281 \mathrm{~nm}$ in the swollen state at $10^{\circ} \mathrm{C}$ and increases from 108 to $141 \mathrm{~nm}$ 
Fig. 3 Scheme of the setup. The NIPAM/BIS solution is pumped through the reactor via syringe pumps. The reaction is initiated by continuous addition of APS solution. The reaction progress is monitored by in situ FTIR.

The reaction solution is quenched in a $p$-methoxyphenol solution, purified by centrifugation and characterized by photon correlation spectroscopy (PCS)

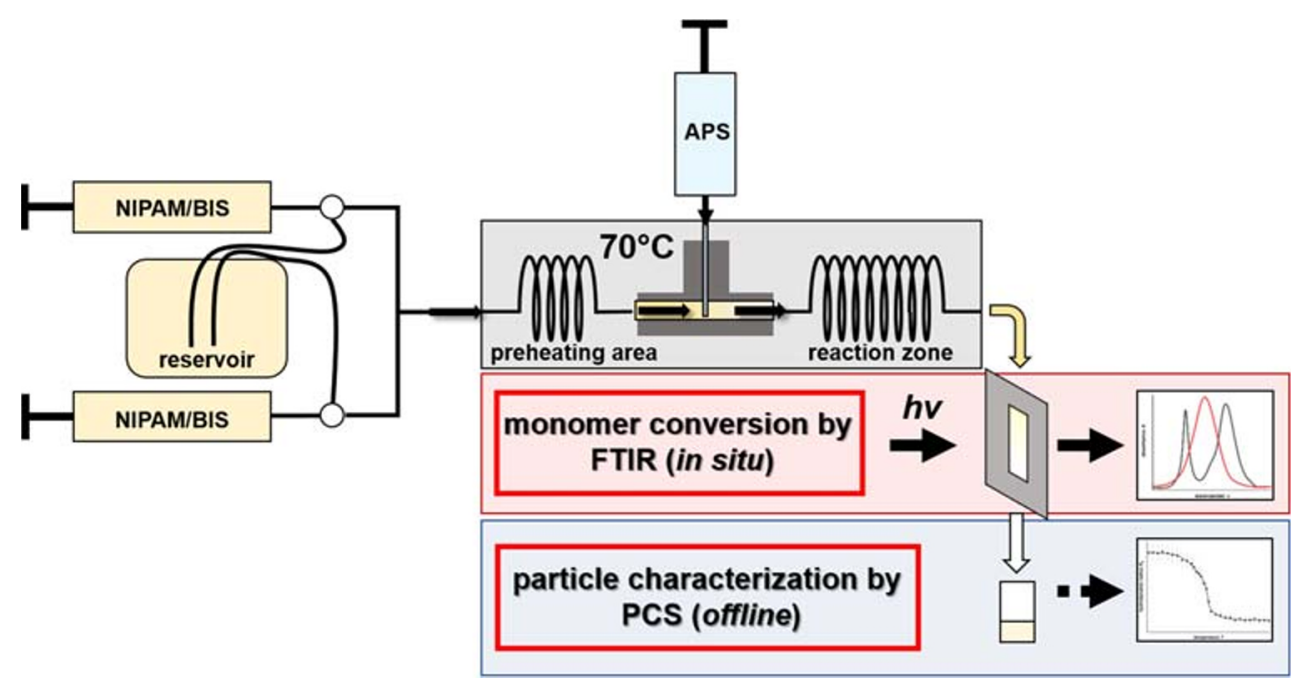

increase in size can be observed, whereas the size difference between $10 \mathrm{~min}$ and $60 \mathrm{~min}$ is rather small. This trend is particularly evident when the corresponding phase images of the microgels (Fig. $5 \mathrm{a}-\mathrm{c}$ ) are considered. In addition to the height images, the particles show a typical core-corona structure in the phase image, becoming more distinct after 10 and $60 \mathrm{~min}$ [61]. The core-corona structure suggests a cross-linker gradient, leading to a distinct fuzzy periphery during the particle growth.

A quantification of the AFM images shows an increase of the particle size. The height and the median of the particle radius increase between $5 \mathrm{~min}$ (height: $10 \mathrm{~nm}$, median radius: $160 \mathrm{~nm}$ ) and $10 \mathrm{~min}$ (height: $35 \mathrm{~nm}$, median radius: $225 \mathrm{~nm}$ ), while the change from 10 to $60 \mathrm{~min}$ (height: $50 \mathrm{~nm}$, median radius: $250 \mathrm{~nm}$ ) is less significant. However, the shape of the particle distribution implies a rather high polydispersity (Fig. 6 a, b). An increased polydispersity in comparison to batch conditions is a common observation in continuous flow experiments, due to a lack of mixing [40]. To quantify the polydispersity of the microgels in their native state, we performed SLS experiments with the purified microgels after a residence time of $60 \mathrm{~min}$ in the collapsed state (Fig. 7).

First a model-independent analysis of the scattering data was performed to gain insights into the structural properties of the microgel particles. To get an idea of the general shape of the microgels, the ratio of the radius of gyration and the hydrodynamic radius $\left(R_{g} / R_{h}\right)$ was investigated. Figure $7 \mathrm{a}$ shows the Guinier analysis at $50{ }^{\circ} \mathrm{C}$. The slope of the plot led to a $R_{g}$ of $109.7 \mathrm{~nm}$ and a $R_{g}$ to $R_{h}$ ratio of 0.778 and shows that the microgels behave like hard spheres in the collapsed state $[62,63]$.

Furthermore, a model-dependent adaption of the data was performed. Figure $7 \mathrm{~b}$ shows a plot of the normalized scattering intensity $I_{\text {norm }}(q)$ against the scattering vector $q$ at $50{ }^{\circ} \mathrm{C}$ between 0.008 and $0.03 \mathrm{~nm}^{-1}$. It is to mention 


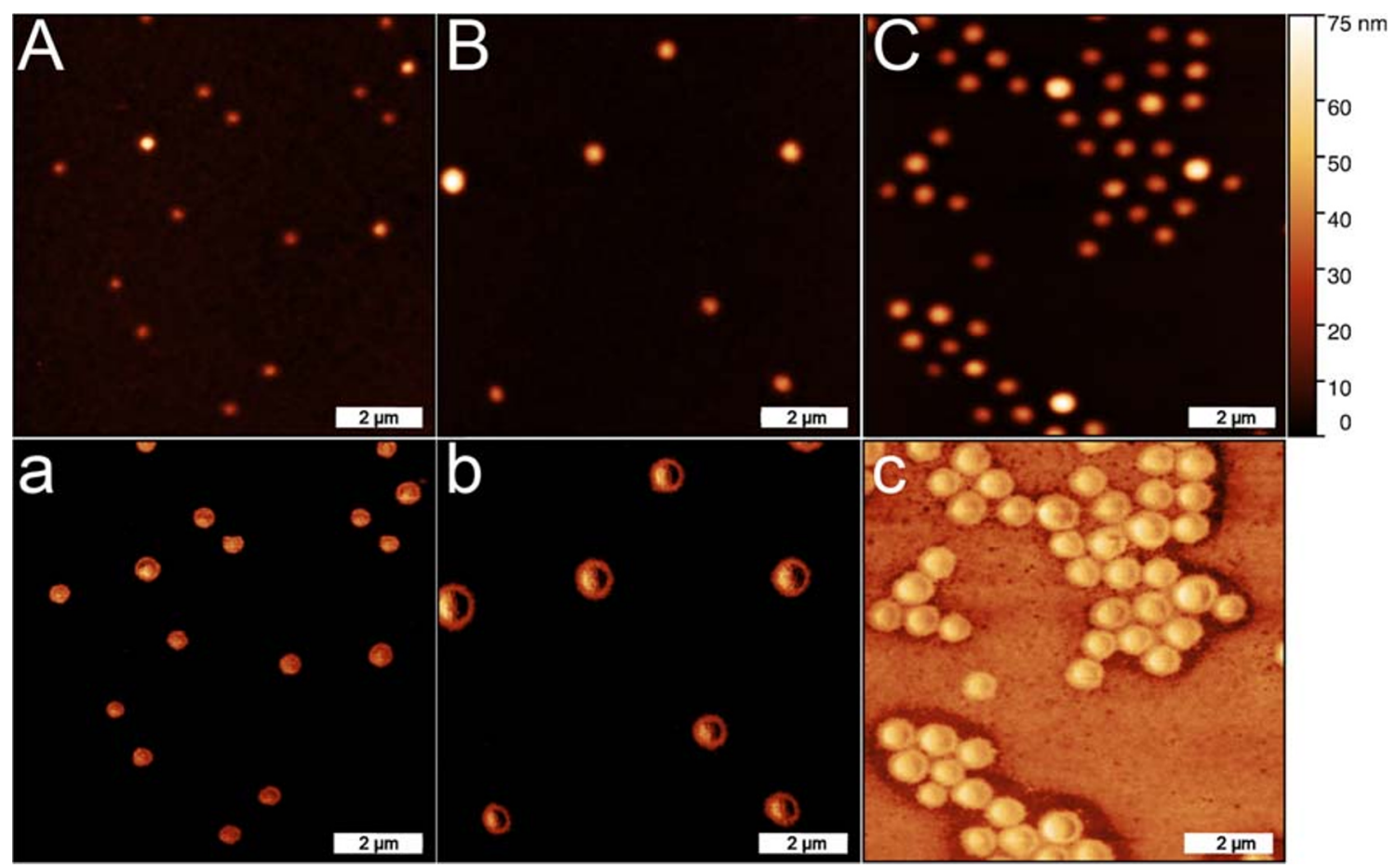

Fig. 5 AFM images of PNIPAM microgels after a residence time of 5 min (A, a), $10 \mathrm{~min}(\mathrm{~B}, \mathrm{~b})$ and 60 min $(\mathrm{C}$, c). All measurements were performed in the dried state at room temperature. The height images are marked with a capital letter and the corresponding phase images with a lower case letter

that the form factor minimum could not be resolved, which makes a hard-sphere analysis more challenging. Nevertheless, we performed a fit of the obtained data using the hard-sphere model. For the collapsed particles, a radius of gyration of $110.0 \mathrm{~nm}$ was obtained with a PDI-value of 1.23. An increased polydispersity is in accordance with observations we made in the dry state using AFM. We assume that the higher polydispersity in our experiments occurs due to the absence of mixing. Therefore, the reaction process is completely driven by diffusion, since turbulent mixing only occurs at high Reynolds numbers, typically under very high flow velocities [64]. A more monodisperse size distribution can be obtained by using a mixing element [65]. Despite the higher polydispersity compared to classically synthesized microgels, we could show by temperature-dependent PCS and AFM that at all studied residence times, microgel particles with the characteristic swelling behavior were obtained with the presented flow reactor.

\section{Kinetics of microgel formation}

In addition to offline investigations of the microgel formation, we analyzed the conversion of the monomers in situ, utilizing FTIR spectroscopy. Therefore, the flow reactor was connected to a flow cell in a FTIR spectrometer. The monomers and the initiator were dissolved in $\mathrm{D}_{2} \mathrm{O}$ to avoid significant absorption of water between 1700 and 1600 $\mathrm{cm}^{-1}$, opening the spectroscopic window to investigate prominent $v(\mathrm{C}=\mathrm{O})$ and $v(\mathrm{C}=\mathrm{C})$ vibrations of microgels and
Fig. 6 Averaged height of the microgel particles obtained from the AFM images (see SI: Fig. 6) at the residence times of $5 \mathrm{~min}$, $10 \mathrm{~min}$ and $60 \mathrm{~min}(\mathbf{a})$ and the corresponding particle distribution (b). The error bars correspond to the standard deviation
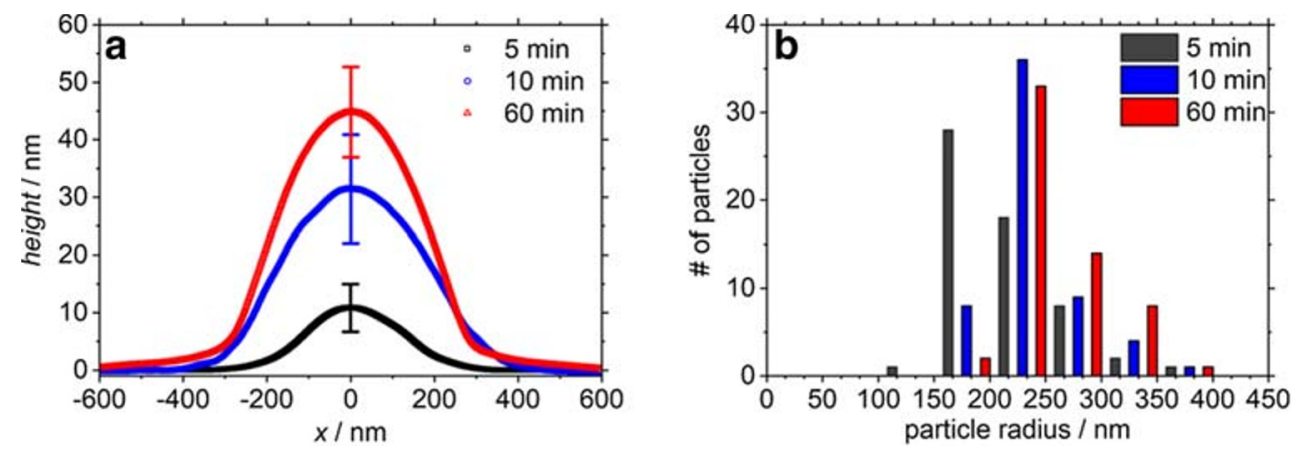
Fig. 7 SLS of microgels obtained after $60 \mathrm{~min}$ in the flow reactor at $50^{\circ} \mathrm{C}$, normalized with toluene. a: Linear fit of the Guinier region of the scattering curve up to a maximum value of $q R_{g}<1$. b: The scattering curve of the collapsed microgels was fitted with a hard-sphere model
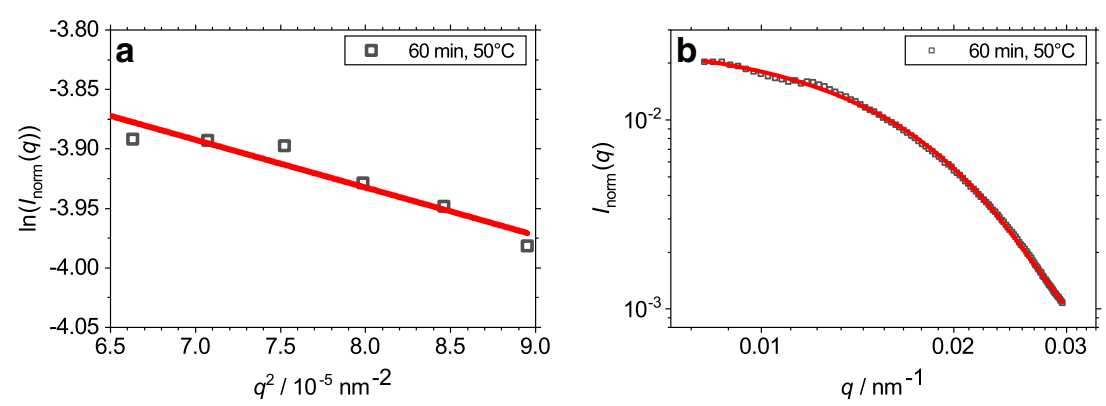

monomers. A flow cell containing two $\mathrm{CaF}_{2}$-windows separated by a PTFE spacer with a thickness of $0.1 \mathrm{~mm}$ and a slit with a width of $2 \mathrm{~mm}$ was installed in the spectrometer. A spectrum of $\mathrm{D}_{2} \mathrm{O}$ was recorded directly before the experiment to compensate solvent absorbance. For reconstruction of the experimental spectra by linear combination, reference spectra of the stock solution and the purified microgel with a residence time of $60 \mathrm{~min}$ were recorded. The corresponding reference solutions were obtained by adding a fixed concentration of $\mathrm{NaSCN}\left(154 \mathrm{mM}\right.$ in $\left.\mathrm{D}_{2} \mathrm{O}\right)$ as a chemical standard to the freeze-dried stock solution (concentration: $97 \mathrm{mM}$ with respect to the initial amount of NIPAM) to verify a constant path length between the reference spectra. The reference solution of the purified microgel was prepared likewise. NaSCN was only used as a chemical standard in reference spectra of the stock solution and the purified microgel, since additives could cause an influence on the reaction by a change in the ionic strength, solvency effects, or quenching. The recorded reference spectra show significant differences in vibrational modes between the stock solution and the purified polymer (Fig. 8a).

Between 1700 and $1350 \mathrm{~cm}^{-1}$ prominent absorption bands are visible for NIPAM and PNIPAM. The bands can be assigned with density functional theory (DFT) (see SI: Fig. 8). For the monomer, characteristic bands of $v(\mathrm{C}=\mathrm{C})$

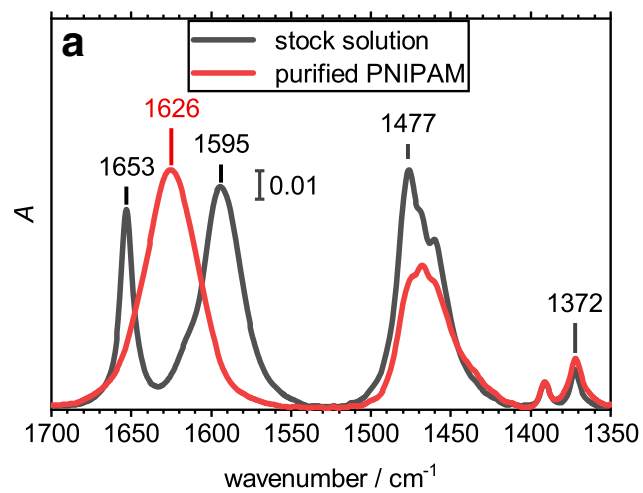

Fig. 8 a: Reference spectra of the stock solution and the purified microgel in $\mathrm{D}_{2} \mathrm{O}$ (97 $\mathrm{mM}$ with respect to the initial amount of NIPAM). NaSCN was used as a chemical standard to verify a constant path length (also see SI: Fig. 7). b: In situ FTIR spectrum of the reaction solution with a residence time of $5 \mathrm{~min}$ inside the reactor (purple, at $1653 \mathrm{~cm}^{-1}$ and $v(\mathrm{C}=\mathrm{O})$ at $1595 \mathrm{~cm}^{-1}$ can be observed. The respective amide I vibration of the polymer is shifted to higher frequencies at $1626 \mathrm{~cm}^{-1}$. The bending vibration of amide II is found at $1477 \mathrm{~cm}^{-1}$, while this vibration overlaps with $\mathrm{CH}_{3}$ bending vibrations in this region [46]. $\delta_{\mathrm{S}}\left(\mathrm{CH}_{3}\right)$ and $\delta_{\mathrm{s}}\left(\mathrm{CH}_{2}\right)$ vibrations from NIPAM are detected at 1392 and $1372 \mathrm{~cm}^{-1}$, as well as $\delta(\mathrm{C}-\mathrm{H})$ deformation of the polymer. This observation is in accordance to literature [46]. The vanishing of $\mathrm{C}=\mathrm{C}$ double bonds is a typical probe for monitoring the progress in precipitation polymerizations [49]. In Fig. 8b an in situ FTIR spectrum of the solution with a residence time of $5 \mathrm{~min}$ inside of the reactor is shown. The spectrum consists of characteristic vibrational bands of the stock solution, as well as overlapping contributions by the polymer. A linear combination (dashed line) of spectra from the stock solution (black) and the purified polymer (red) is in good accordance to the experimental data (purple, solid line).

With increasing residence time, the amide I vibration of the polymer at $1626 \mathrm{~cm}^{-1}$ increases, whereas the intensity of the prominent monomer vibrations at 1653 and $1595 \mathrm{~cm}^{-1}$ vanishes (Fig. 9). After $60 \mathrm{~min}$, no significant contribution by the stock solution is observable. For all residence times, the reconstruction is in good accordance to the experimental data. To quantify the conversion Eq. 7 was used, containing

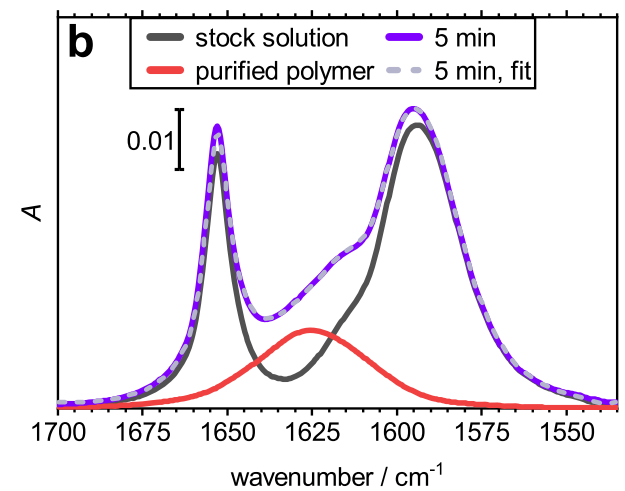

solid line) and corresponding linear combination (dashed line). The reference spectrum of the stock solution (black) and the purified polymer (red) are scaled according to their contribution to the linear combination 


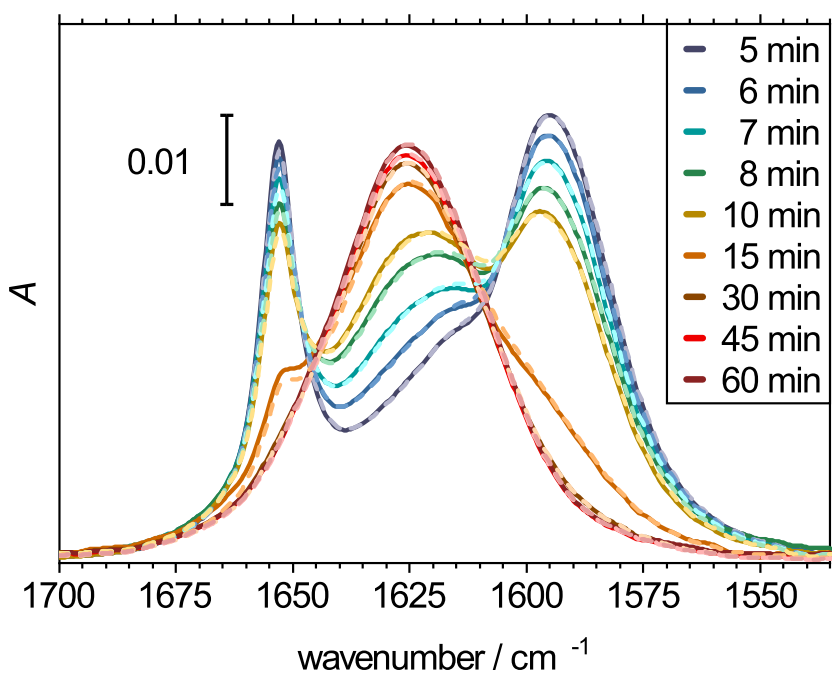

Fig. 9 In situ FTIR spectra of the reaction solutions after residence times between 5 and 60 min inside the reactor and corresponding linear combinations (dashed lines) from the reference spectra of the stock solution and the purified polymer (for raw and corrected spectra see SI: Fig. 9, 10)

the determined linear combination coefficients for the stock solution $a_{s}$ and the purified polymer $a_{p}$.

$X=\frac{a_{p}}{a_{s}+a_{p}}$

The relative conversion increases rapidly within the first minutes after initiation (Fig. 10, red). Five minutes after leaving the reactor, $21 \%$ of the stock solution is converted. After $15 \mathrm{~min}$, over $80 \%$ and after $60 \mathrm{~min}, 98 \%$ of the monomer reacted to polymer. High conversions at $70^{\circ} \mathrm{C}$ within the first $15 \mathrm{~min}$ can also be seen under batch conditions [51].

In addition to the conversion, the volume of the collapsed microgels at different residence times is shown in Fig. 10. It can be seen that the volume increases rapidly within the

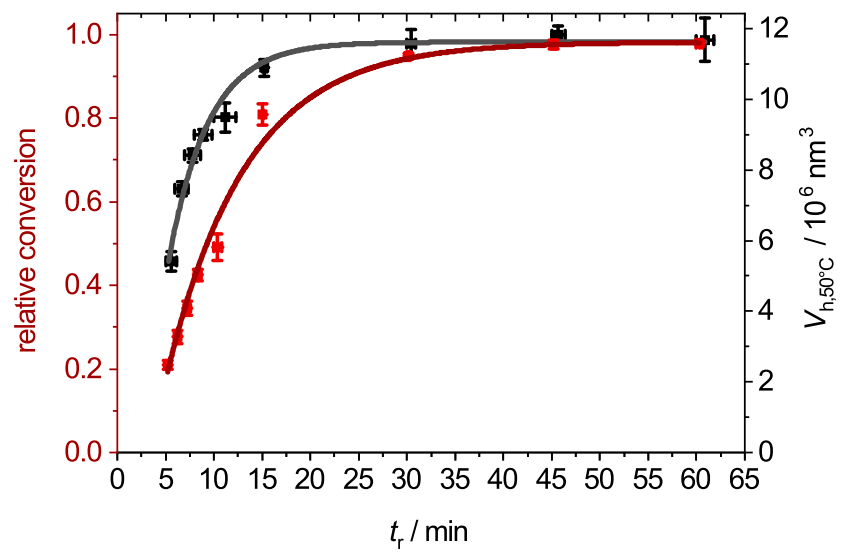

Fig. 10 Relative monomer conversion calculated from linear combinations of in situ FTIR experiments (red) and corresponding particle

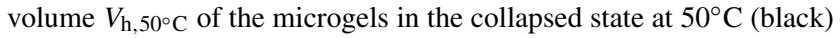
against the residence time $t_{\mathrm{r}}$ with a single exponential approach (Eq. 7) the first $15 \mathrm{~min}$. After about $30 \mathrm{~min}$ no significant increase in the particle size is observed. The data points in Fig. 10 are described with Eq. 8, where $X_{\max }$ is the maximum conversion, $t$ the time, $t_{0}$ the offset from the origin and $k$ the rate constant.

$X=X_{\max }-X_{\max } \exp \left(-\left(t-t_{o}\right) k\right)$

With the single exponential approach, we assume that the rather small amount of cross-linker does not significantly influence the kinetics of the conversion and the particle growth. The particle volume is fitted with the same equation, whereby $X_{\max }$ is replaced by the maximum of the hydrodynamic volume $V_{\mathrm{h}, \max }$. The results are shown in Table 2.

A linear dependency between the monomer conversion and a volume increase in the collapsed state was already shown by $\mathrm{Wu}$ et al. [51], when all reacted polymer ends up in the microgel particles. In contrast to that, the values of the kinetics in Table 2 show a deviation between the rate constants of the conversion $\left(k_{X}=0.12 \mathrm{~min}^{-1}\right)$ and the particle growth $\left(k_{V}=0.24 \mathrm{~min}^{-1}\right)$. It has to be mentioned that the effective concentration of polymerized NIPAM in the reference solution of the purified polymer might be overestimated, since the purification process could have changed the substance composition, compared to the initial amount. A gravimetric analysis shows that the influence on the conversion kinetics due to a loss of NIPAM is not significant, compared to deviations between the particle growth and the conversion (see SI: Fig. 11). The deviation between the conversion and the particle growth kinetics might occur due to reacted monomer not being built into microgel particles, but as a part of oligomers. The absolute values of the rate constants and the total conversion strongly depend on the respective reaction conditions and can be influenced by the monomer properties [66], the addition of accelerators [50], or by surfactants [40]. Furthermore, the progress implies an offset from the origin, indicating the existence of an induction period which could not be accessed here. The offset and the deviation between the rate constants most likely originates from a lack of mixing, leading to laminar flows and a diffusion-driven reaction

Table 2 Fitting parameters from a single exponential approach (8) of the kinetic data in Fig. 10

\begin{tabular}{lllll}
\hline & $\begin{array}{l}X_{\max } \\
(\%)\end{array}$ & $\begin{array}{l}V_{\mathrm{h}, \max } \\
\left(10^{6} \mathrm{~nm}^{3}\right)\end{array}$ & $\begin{array}{l}t_{0} \\
(\mathrm{~min})\end{array}$ & $\begin{array}{l}k \\
\left(\mathrm{~min}^{-1}\right)\end{array}$ \\
\hline rel. conv. & $98.2 \pm 0.1$ & & $3.4 \pm 0.3$ & $0.12 \pm 0.01$ \\
$V_{\mathrm{h}, 50^{\circ} \mathrm{C}}$ & & $11.6 \pm 0.2$ & $2.7 \pm 0.4$ & $0.24 \pm 0.03$ \\
\hline
\end{tabular}

The parameters are described by the maximum conversion $X_{\max }$, the volume in the fully grown, collapsed state $V_{\mathrm{h} \text {,max }}$, the offset with respect to the origin $t_{0}$ and the rate constant $k$ 
process. Under these circumstances, the slower conversion kinetics might originate from a certain amount of time the initiator radicals need to diffuse through the solution. This might lead to further monomer conversion over time, in which the formed oligomers are not built in the microgels. The in situ experiments reveal that mixing not only plays a crucial role in the context of polydispersity [40], but might also have an important influence on the incorporation of monomers and oligomers in microgel particles and will be of great interest in future experiments.

\section{Conclusion}

In this work, we established a continuous flow reactor with a preheating area for continuous synthesis of thermoresponsive microgels with simultaneous monitoring of the reaction progress. FTIR spectroscopy was used as it represents a non-invasive method to study the monomer conversion in an acrylamide-based precipitation polymerization, over an arbitrary period of time. Characteristic vibrations of the monomer and the polymer were used as probes to study the progress on a molecular level. Investigations revealed that the experimental spectra can be described by a linear combination of reference spectra obtained from the stock solution and the polymer between 1710 and $1530 \mathrm{~cm}^{-1}$. As the rate constants of the conversion and the growth kinetics differ from each other, the experiments imply a crucial role of mixing not only in the context of polydispersity, but also in terms of particle growth. The implementation of mixing devices will be an important aspect in upcoming experiments to decrease polydispersity and to optimize microgel formation. The setup allowed to realize reaction times between 5 and $60 \mathrm{~min}$ which was used to determine the particle growth rate of $0.24 \mathrm{~min}^{-1}$. From the FTIR data we obtained a rate of $0.12 \mathrm{~min}^{-1}$. The results show that mixing not only has a major influence on polydispersity in continuous flow setups but also seems to influence the incorporation of oligomers in the microgels. Furthermore, continuous flow setups with essentially unlimited reservoirs in combination with $\mathrm{x}$-ray or neutron scattering experiments can open the gate for automatic screening of reaction parameters in microgel syntheses and long term studies of early stages in the reaction progress.

Acknowledgements The authors gratefully acknowledge Ina Ehring for the purification of the monomer. We thank Andreas Helfmann, Jan-Ulrich Klamert, Leo Alexander Walters, Manfred Hoffmann and everyone from the mechanical workshop at Bielefeld University for the fabrication of the flow reactor, presented in this work. Viktor Sabadasch, Lukas Gött-Zink and Jorge Esteban Vázquez are acknowledged for very constructive and fruitful discussions concerning microgel syntheses, FTIR spectroscopy and static light scattering.
Funding Open Access funding enabled and organized by Projekt DEAL.

\section{Compliance with ethical standards}

Conflict of interest The authors declare that they have no conflict of interest.

Open Access This article is licensed under a Creative Commons Attribution 4.0 International License, which permits use, sharing, adaptation, distribution and reproduction in any medium or format, as long as you give appropriate credit to the original author(s) and the source, provide a link to the Creative Commons licence, and indicate if changes were made. The images or other third party material in this article are included in the article's Creative Commons licence, unless indicated otherwise in a credit line to the material. If material is not included in the article's Creative Commons licence and your intended use is not permitted by statutory regulation or exceeds the permitted use, you will need to obtain permission directly from the copyright holder. To view a copy of this licence, visit http:// creativecommonshorg/licenses/by/4.0/.

\section{References}

1. Murray MJ, Snowden MJ (1995) The preparation, characterisation and applications of colloidal microgels. Adv Colloid Interfac 54:73-91. https://doi.org/10.1016/0001-8686(94)00222-X

2. Saunders BR, Vincent B (1999) Microgel particles as model colloids: theory, properties and applications. Adv Colloid Interfac 80:1-25. https://doi.org/10.1016/S0001-8686(98)00071-2

3. Pelton R (2000) Temperature-sensitive aqueous microgels. Adv Colloid Interfac 85:1-33. https://doi.org/10.1016/S00018686(99)00023-8

4. Pich A, Richtering W (2011) Chemical design of responsive microgels. Adv. Polym. Sci. Springer, Berlin. https://doi.org/10.1007/978-3-642-16379-1

5. Karg M, Pich A, Hellweg T, Hoare T, Lyon LA, Crassous JJ, Suzuki D, Gumerov RA, Schneider S, Potemkin II, Richtering W (2019) Nanogels and microgels: from model colloids to applications, recent developments, and future trends. Langmuir 35:6231-6255. https://doi.org/10.1021/acs.langmuir.8b04304

6. Liu R, Fraylich M, Saunders BR (2009) Thermoresponsive copolymers: from fundamental studies to applications. Colloid Polym Sci 287:627-643. https://doi.org/10.1007/s00396-0092028-x

7. Hoare T, Pelton R (2004) Highly $\mathrm{pH}$ and temperature responsive microgels functionalized with vinylacetic acid. Macromolecules 37:2544-2550. https://doi.org/10.1021/ma035658m

8. Wrede O, Reimann Y, Lülsdorf S, Emmrich D, Schneider K, Schmid A. J., Zauser D, Hannappel Y, Beyer A, Schweins R, Gölzhäuser A, Hellweg T, Sottmann T (2018) Volume phase transition kinetics of smart $\mathrm{N}$-n-propylacrylamide microgels studied by time-resolved pressure jump small angle neutron scattering. Sci Rep-UK 8:13781. https://doi.org/10.1038/s41598-018-31976-4

9. Backes S, Krause P, Tabaka W, Witt MU, von Klitzing R (2017) Combined cononsolvency and temperature effects on adsorbed PNIPAM microgels. Langmuir 33:14269-14277. https://doi.org/10.1021/acs.langmuir.7b02903

10. Karg M, Pastoriza-Santos I, Rodriguez-González B, von Klitzing $\mathrm{R}$, Wellert S, Hellweg T (2008) Temperature, $\mathrm{pH}$, and ionic strength induced changes of the swelling behavior of PNIPAMpoly(allylacetic acid) copolymer microgels. Langmuir 24:63006306. https://doi.org/10.1021/la702996p 
11. Sabadasch V, Wiehemeier L, Kottke T, Hellweg T (2020) Core-shell microgels as thermoresponsive carriers for catalytic palladium nanoparticles. Soft Matter 16:5422-5430. https://doi.org/10.1039/D0SM00433B

12. Kleinschmidt D, Fernandes MS, Mork M, Meyer AA, Krischel J, Anakhov MV, Gumerov RA, Potemkin II, Rueping M, Pich A (2020) Enhanced catalyst performance through compartmentalization exemplified by colloidal 1-proline modified microgel catalysts. J Colloid Interf Sci 559:76-87. https://doi.org/10.1016/j.jcis.2019.10.005

13. Welsch N, Wittemann A, Ballauff M (2009) Enhanced activity of enzymes immobilized in thermoresponsive core-shell microgels. J Phys Chem B 113:16039-16045. https://doi.org/10.1021/ jp907508w

14. Schachschal S, Adler HJ, Pich A, Wetzel S, Matura A, van Pee KH (2011) Encapsulation of enzymes in microgels by polymerization/cross-linking in aqueous droplets. Colloid Polym Sci 289:693-698. https://doi.org/10.1007/s00396-011-2392-1

15. Debord JD, Lyon LA (2000) Thermoresponsive photonic crystals. J Phys Chem B 104:6327-6331. https://doi.org/10.1021/ jp001238c

16. Karg M, Lu Y, Carbó-Argibay E, Pastoriza-Santos I, Pérez-Juste J, Liz-Marzán LM, Hellweg T (2009) Multiresponsive hybrid colloids based on gold nanorods and poly(NIPAM-co-allylacetic acid) microgels: temperature- and $\mathrm{pH}$-tunable plasmon resonance. Langmuir 25:3163-3167. https://doi.org/10.1021/la803458j

17. Döring A, Birnbaum W, Kuckling D (2013) Responsive hydrogels-structurally and dimensionally optimized smart frameworks for applications in catalysis, micro-system technology and material science. Chem Soc Rev 42:7391-7420. https://doi.org/10.1039/C3CS60031A

18. Islam MR, Gao Y, Li X, Serpe MJ (2014) Responsive polymers for biosensing and protein delivery. J Mater Chem B 2:2444-2451. https://doi.org/10.1039/c3tb21657h

19. Dirksen M, Dargel C, Meier L, Brändel T, Hellweg T (2020) Smart microgels as drug delivery vehicles for the natural drug aescin: uptake, release and interactions. Colloid Polym Sci 298:505-518. https://doi.org/10.1007/s00396-020-04632-5

20. Schmaljohann D (2006) Thermo- and pH-responsive polymers in drug delivery. Adv Drug Deliver Rev 58:1655-1670. https://doi.org/10.1016/j.addr.2006.09.020

21. Zeiser M, Freudensprung I, Hellweg T (2012) Linearly thermoresponsive core-shell microgels: Towards a new class of nanoactuators. Polymer 53:6096-6101. https://doi.org/10.1016/j.polymer. 2012.10.001

22. Ito $\mathrm{T}$, Hioki $\mathrm{T}$, Yamaguchi $\mathrm{T}$, Shinbo $\mathrm{T}, \mathrm{Si}$ Nakao, Kimura $S$ (2002) Development of a molecular recognition ion gating membrane and estimation of its pore size control. J Am Chem Soc 124:7840-7846. https://doi.org/10.1021/ja012648x

23. Frost S, Ulbricht M (2013) Thermoresponsive ultrafiltration membranes for the switchable permeation and fractionation of nanoparticles. J Membrane Sci 448:1-11. https://doi.org/10.1016/ j.memsci.2013.07.036

24. Serpe MJ, Jones CD, Lyon LA (2003) Layer-by-layer deposition of thermoresponsive microgel thin films. Langmuir 19:87598764. https://doi.org/10.1021/la034391h

25. Uhlig K, Wegener T, He J, Zeiser M, Bookhold J, Dewald I, Godino N, Jaeger M, Hellweg T, Fery A, Duschl C (2016) Patterned thermoresponsive microgel coatings for noninvasive processing of adherent cells. Biomacromolecules 17:1110-1116. https://doi.org/10.1021/acs.biomac.5b01728

26. Seiffert S, Weitz DA (2010) Controlled fabrication of polymer microgels by polymer-analogous gelation in droplet microfluidics. Soft Matter 6:3184. https://doi.org/10.1039/c0sm00071j
27. Thiele J, Seiffert S (2011) Double emulsions with controlled morphology by microgel scaffolding. Lab Chip 11:3188-3192. https://doi.org/10.1039/c1lc20242a

28. Heida T, Neubauer JW, Seuss M, Hauck N, Thiele J, Fery A (2017) Mechanically defined microgels by droplet microfluidics. Macromol Chem Phys 218:1600418. https://doi.org/10.1002/ macp. 201600418

29. Hertle Y, Hellweg T (2013) Thermoresponsive copolymer microgels. J Mater Chem B 1:5874-5885. https://doi.org/10.1039/ c3tb21143f

30. Berndt I, Richtering W (2003) Doubly temperature sensitive Core-Shell microgels. Macromolecules 36:8780-8785. https://doi.org/10.1021/ma034771+

31. Karg M, Wellert S, Prevost S, Schweins R, Dewhurst C, LizMarzán LM, Hellweg T (2011) Well defined hybrid PNIPAM core-shell microgels: size variation of the silica nanoparticle core. Colloid Polym Sci 289:699-709. https://doi.org/10.1007/s00396010-2327-2

32. Richtering W, Saunders BR (2014) Gel architectures and their complexity. Soft Matter 10:3695-3702. https://doi.org/10.1039/ c4sm00208c

33. Geisel K, Rudov AA, Potemkin II, Richtering W (2015) Hollow and Core-Shell microgels at Oil-Water interfaces: spreading of soft particles reduces the compressibility of the monolayer. Langmuir 31:13145-13154. https://doi.org/10.1021/acs.langmuir. 5 b03530

34. Nayak S, Lyon LA (2005) Weiche Nanotechnologie mit weichen Nanopartikeln. Angew Chem 117:7862-7886. https://doi.org/10.1002/ange.200501321

35. Goodwin JW, Ottewill RH, Pelton R, Vianello G, Yates DE (1978) Control of particle size in the formation of polymer latices. Brit Polym J 10:173-180. https://doi.org/10.1002/pi.4980100304

36. Loxley A, Vincent B (1997) Equilibrium and kinetic aspects of the $\mathrm{pH}$-dependent swelling of poly(2-vinylpyridine-co-styrene) microgels. Colloid Polym Sci 275:1108-1114. https://doi.org/10. 1007/s003960050190

37. Jones CD, Lyon LA (2003) Shell-restricted swelling and core compression in poly(N-isopropylacrylamide) core-shell microgels. Macromolecules 36:1988-1993. https://doi.org/10.1021/ $\mathrm{ma} 021079 \mathrm{q}$

38. Pelton RH, Chibante P (1986) Preparation of aqueous latices with N-isopropylacrylamide. Colloid Surface 20:247-256. https://doi.org/10.1016/0166-6622(86)80274-8

39. Li W, Greener J, Voicu D, Kumacheva E (2009) Multiple modular microfluidic $(\mathrm{m} 3)$ reactors for the synthesis of polymer particles. Lab on a chip 9:2715-2721. https://doi.org/10.1039/b906626h

40. Wolff HJM, Kather M, Breisig H, Richtering W, Pich A, Wessling M (2018) From batch to continuous precipitation polymerization of thermoresponsive microgels. ACS Appl Mater Inter 10:2479924806. https://doi.org/10.1021/acsami.8b06920

41. Yadav AK, Barandiaran MJ, de La Cal JC (2012) Synthesis of water-borne polymer nanoparticles in a continuous microreactor. Journal Chem Eng J 198-199:191-200. https://doi.org/10.1016/ j.cej.2012.05.091

42. Russum JP, Jones CW, Schork FJ (2004) Continuous reversible addition-fragmentation chain transfer polymerization in miniemulsion utilizing a Multi-Tube reaction system. Macromol Rapid Comm 25:1064-1068. https://doi.org/10.1002/marc. 200400086

43. Keerl M, Smirnovas V, Winter R, Richtering W (2008) Interplay between hydrogen bonding and macromolecular architecture leading to unusual phase behavior in thermosensitive microgels. Angew Chem 120:344-347. https://doi.org/10.1002/ange. 200703728 
44. Keerl M, Smirnovas V, Winter R, Richtering W (2008) Copolymer microgels from mono- and disubstituted acrylamides: phase behavior and hydrogen bonds. Macromolecules 41:6830-6836. https://doi.org/10.1021/ma800785w

45. Wiehemeier L, Cors M, Wrede O, Oberdisse J, Hellweg T, Kottke $\mathrm{T}$ (2019) Swelling behaviour of core-shell microgels in H2O, analysed by temperature-dependent FTIR spectroscopy. Phys Chem Chem Phys 21:572-580. https://doi.org/10.1039/C8CP05911J

46. Futscher MH, Philipp M, Müller-Buschbaum P, Schulte A (2017) The role of backbone hydration of poly( $\mathrm{N}$-isopropyl acrylamide) across the volume phase transition compared to its monomer. Sci Rep-UK 7:17012. https://doi.org/10.1038/s41598-017-17272-7

47. Maeda Y, Higuchi T, Ikeda I (2000) Change in hydration state during the coil-globule transition of aqueous solutions of poly $(\mathrm{N}$ -isopropylacrylamide) as evidenced by FTIR spectroscopy. Langmuir 16:7503-7509. https://doi.org/10.1021/la0001575

48. Maeda Y, Nakamura T, Ikeda I (2002) Change in solvation of poly ( N,N-diethylacrylamide) during phase transition in aqueous solutions as observed by IR spectroscopy. Macromolecules 35:10172-10177. https://doi.org/10.1021/ma020945w

49. Meyer-Kirschner J, Kather M, Pich A, Engel D, Marquardt W, Viell J, Mitsos A (2016) In-line monitoring of monomer and polymer content during microgel synthesis using precipitation polymerization via raman spectroscopy and indirect hard modeling. Appl Spectrosc 70:416-426. https://doi.org/10.1177/ 0003702815626663

50. Virtanen OLJ, Kather M, Meyer-Kirschner J, Melle A, Radulescu A, Viell J, Mitsos A, Pich A, Richtering W (2019) Direct monitoring of microgel formation during precipitation polymerization of N-isopropylacrylamide using in situ SANS. ACS Omega 4:36903699. https://doi.org/10.1021/acsomega.8b03461,

51. Wu X, Pelton RH, Hamielec AE, Woods DR, McPhee W (1994) The kinetics of poly(N-isopropylacrylamide) microgel latex formation. Colloid Polym Sci 272:467-477. https://doi.org/10.1007/ BF00659460

52. Acciaro R, Gilányi T, Varga I (2011) Preparation of monodisperse poly(N-isopropylacrylamide) microgel particles with homogenous cross-link density distribution. Langmuir 27:7917-7925. https://doi.org/10.1021/la2010387

53. Provencher SW (1982) CONTIN: A general purpose constrained regularization program for inverting noisy linear algebraic and integral equations. Comput Phys Commun 27:229-242. https://doi.org/10.1016/0010-4655(82)90174-6

54. Guinier A, Fournet G (1956) Small angle scattering of X-rays. Wiley, New York. https://doi.org/10.1002/pol.1956.120199326
55. Nečas D, Klapetek P (2012) Gwyddion: an open-source software for SPM data analysis. Open Phys 10. https://doi.org/10.2478/ s11534-011-0096-2

56. Schneider CA, Rasband WS, Eliceiri KW (2012) NIH Image to ImageJ: 25 years of image analysis. Nat Methods 9:671-675. https://doi.org/10.1038/nmeth.2089

57. Frisch MJ, Trucks GW, Schlegel HB et al (2004) Gaussian 03, Revision D.01. Wallingford CT

58. dos Santos WN, Iguchi CY, Gregorio R (2008) Thermal properties of poly(vinilidene fluoride) in the temperature range from 25 to $210^{\circ}$ C. Polym Test 27:204-208. https://doi.org/10.1016/j. polymertesting.2007.10.005

59. Peet MJ, Hasan HS, Bhadeshia H (2011) Prediction of thermal conductivity of steel. Int J Heat Mass Tran 54:2602-2608. https://doi.org/10.1016/j.ijheatmasstransfer.2011.01.025

60. Wedel B, Hertle Y, Wrede O, Bookhold J, Hellweg T (2016) Smart homopolymer microgels: influence of the monomer structure on the particle properties. Polymers 8. https://doi.org/10.3390/ polym8040162

61. Hoppe Alvarez L, Eisold S, Gumerov RA, Strauch M, Rudov AA, Lenssen P, Merhof D, Potemkin II, Simon U, Wöll D (2019) Deformation of microgels at solid-liquid interfaces visualized in three-dimension. Nano Lett 19:8862-8867. https://doi.org/10.1021/acs.nanolett.9b03688

62. Senff H, Richtering W (2000) Influence of cross-link density on rheological properties of temperature-sensitive microgel suspensions. Colloid Polym Sci 278:830-840. https://doi.org/10.1007/ s003960000329

63. Pyett S, Richtering W (2005) Structures and dynamics of thermosensitive microgel suspensions studied with three-dimensional cross-correlated light scattering. J Chem Phys 122:34709. https://doi.org/10.1063/1.1834492

64. Schulte TH, Bardell RL, Weigl BH (2002) Microfluidic technologies in clinical diagnostics. Clin Chim Acta 321:1-10. https://doi.org/10.1016/S0009-8981(02)00093-1

65. Lueth FG, Pauer W, Moritz HU (2013) Properties of smart-scaled PTFE-tubular reactors for continuous emulsion polymerization reactions. Macromol Sy 333:69-79. https://doi.org/10.1002/masy. 201300086

66. Janssen FAL, Kather M, Kröger LC, Mhamdi A, Leonhard K, Pich A, Mitsos A (2017) Synthesis of poly(N-vinylcaprolactam)-based microgels by precipitation polymerization: process modeling and experimental validation. Ind Eng Chem Res 56:14545-14556. https://doi.org/10.1021/acs.iecr.7b03263

Publisher's note Springer Nature remains neutral with regard to jurisdictional claims in published maps and institutional affiliations. 